\title{
Implementing the European Code of Cancer Practice to Improve Europe's Cancer Outcomes
}

\author{
Mark Lawler, ${ }^{1}$ Kathy Oliver, ${ }^{2}$ Stefan Gijssels, ${ }^{3}$ Matti Aapro, ${ }^{4}$ Agnese Abolina, ${ }^{4}$ Mike Morrissey, ${ }^{4}$ Richard Price ${ }^{4}$ and \\ Peter Selby 5,6 \\ 1. Faculty of Medicine, Health and Life Sciences, Patrick G Johnston Centre for Cancer Research, Queen's University Belfast, UK; \\ 2. International Brain Tumour Alliance, Tadworth, Surrey, UK; 3. Digestive Cancers Europe, Brussels, Belgium; 4. European Cancer \\ Organisation, Brussels, Belgium; 5. Leeds Institute of Medical Research, University of Leeds and Leeds Teaching Hospitals NHS Trust, Leeds, \\ UK; 6. University of Lincoln, UK
}

DOI: https://doi.org/10.17925/OHR.2021.17.1.9

T he European Code of Cancer Practice, co-produced by cancer professionals, patients and patient advocates, describes patientcentred good clinical cancer practice aimed at improving patient outcomes. There are 10 patient rights supported by a non-technical explanation; three questions that a patient may ask healthcare professionals; and a comprehensive evidence review, which will empower patients and influence healthcare professionals and policymakers. It aims to bridge the gap between cancer guidelines and plans, and the everyday experience of patients and their carers.

\section{Keywords}

European Code, good cancer practice

Disclosure: Mark Lawler, Kathy Oliver, Stefan Gijssels, Matti Aapro, Agnese Abolina, Mike Morrissey, Richard Price and Peter Selby have nothing to disclose in relation to this article.

Acknowledgements: The authors would like to thank all of their co-authors and colleagues, both the patient advocates and the cancer professionals who contributed to the preparation and publication of the European code of Cancer Practice, especially lan Banks for his contribution and encouragement. Thanks are also due to the staff of the European Cancer Organisation and the European Cancer Concord for their tireless work to bring the code and its predecessor Bill of Rights into existence.

Review Process: Double-blind peer review.

Authorship: The named authors meet the International Committee of Medical Journal Editors (ICMJE) criteria for authorship of this manuscript, take responsibility for the integrity of the work as a whole and have given final approval for the version to be published. Mark Lawler, Kathy Oliver, Stefan Gijssels and Peter Selby contributed equally to this work.

Received: 18 February 2021

Accepted: 14 May 2021

Published Online: 6 July 2021

Citation: touchREVIEWS in Oncology \& Haematology. 2021;17(1): Online ahead of journal publication

Corresponding author: Peter Selby, Level 4 , Bexley Wing, St James's University Hospital,

Beckett Street, Leeds LS9 7TF, UK

E: p.j.selby@leeds.ac.uk

Support: No funding was received for

the publication of this article.

\section{The European Code of Cancer Practice}

The European Code of Cancer Practice is a patient-centred manifesto of the key features required to deliver good clinical cancer practice and improve patient outcomes in Europe. ${ }^{1,2}$ It builds upon the European Cancer Patient's Bill of Rights, which was launched in the European Parliament on World Cancer Day 2014 and received the prestigious 2018 European Health Award. ${ }^{3}$ The Code consists of 10 patient rights, with each right supported by a short, non-technical explanation. Three questions are provided which are suggestions for how a patient, in consultation with their oncologist, might discuss their proposed treatment and care programme. ${ }^{1,2}$ The Code is supported by a Medical Literature and Evidence Paper., ${ }^{1,2}$ The goal of the Code is twofold:

- to inform and empower patients to seek excellence in their cancer care

- to directly and indirectly influence healthcare professionals and policy-makers to deliver stateof-the-art clinical cancer practice.

The code is also a response to the broadly varied and unacceptable disparities in the quality of cancer care found across the countries, regions, hospitals and communities of Europe. ${ }^{12,4}$ Specifically, it seeks to promote changes that will improve outcomes of all European cancer patients towards the aim of a 70\% survival with improved quality of life by 2035 . The details of the code initiative and the evidence base have been previously published.2

The European Code of Cancer Practice has a number of distinguishing features.

- It has been systematically co-produced by an equally balanced group of cancer patient advocates and cancer professionals.

- The format is designed to build a bridge between clinical guidelines, healthcare service plans and the everyday experiences of patients.

- Although prepared to be accessible to a wide non-technical audience, it is robustly evidencebased with a comprehensive review of the medical literature and on-going research predicted to improve cancer outcomes in the coming years.

- The process of co-production and the code itself emphasize the importance of patient empowerment.

The following are the 10 key recommendations and rights in the Code.

1. You have a right to equal access to affordable and optimal available cancer care, including the right to a second opinion. The Code ${ }^{1,2}$ outlines the Medical Literature and Evidence that not only characterizes good clinical cancer practice but also highlights substantial disparities between and within countries, regions, hospitals and communities across Europe in the quality of care and the resulting outcomes for cancer patients. Patients are encouraged 
to enquire in their consultations whether the hospitals or cancer centres providing their care deliver state-of-the-art quality clinical cancer practice as defined by guidelines and service specifications, and whether their outcomes are at the national or international standard. ${ }^{1,25-7}$

2. You have a right to information about your own disease and treatment from your medical team and other reliable sources, including patient and professional organizations. Although there have been improvements in communication skills for healthcare professionals including oncologists, this still remains a challenging area with considerable variations across Europe. The code emphasizes the patient's right to clear and empathic communication. It highlights specific recommendations for how a patient may prepare for a medical consultation and how a cancer professional can prepare to communicate effectively, transparently and compassionately with patients and caregivers. ${ }^{1,2,8}$

3. You have a right to information about the quality and safety of care, the level of expertise and the outcomes achieved for your type of cancer in the cancer care service where you are being treated. In addition to information about their own specific cancer, patients also need information about the quality of the cancer service providing their treatment. This information is also essential for healthcare professionals planning to improve their practice, and for health service managers and policy-makers. Information on the quality of cancer services and outcomes for different services and hospitals is usually provided by national level organizations.

4. You have a right to receive care from a specialized multidisciplinary team, ideally as part of a cancer care network. State-of-the-art cancer care is best delivered by a specialized multidisciplinary team, bringing together all the expertise from a wide range of disciplines that contribute to optimal, modern cancer care in an organized and carefully planned way. This approach improves patient outcomes but has to be very actively and carefully developed and delivered. For some patients with rare cancers and for some complex treatments, a degree of centralization of services into larger main general hospitals or cancer centres is appropriate. But these need to be linked carefully to the wider healthcare system in networks such as the Comprehensive Cancer Care Networks described and evidenced by the European Union Member State Joint Action for Cancer Control. ${ }^{9,10}$

5. You have a right to participate in shared decision-making (SDM) with your healthcare team about all aspects of your treatment and care. Cancer care delivery includes a wide range of challenging decision-making which should be tailored to suit individual patients. However, while in SDM the professional lays out the options and recommendations of their multidisciplinary team, the decision is taken by the patient in consultation with the professional. This is widely regarded as the most satisfactory approach for the majority of patients. SDM reduces the regret that patients may experience if they do not have input into major decisions about their care. SDM can be supported by specific decision aid tools. ${ }^{8}$

6. You have a right to be informed about on-going research relevant to you, and your ability and eligibility to participate in research. Cancer research leads to substantial improvements in outcomes for patients, including improved patient experience and quality of life. Continuing research requires active translation into the clinic and the healthcare systems. ${ }^{1,2}$ Clinical trials are vitally important to identify new treatments or diagnostic strategies. There is substantial evidence that the patients who are treated in researchactive hospitals have better outcomes than those who are not. ${ }^{11}$
7. You have a right to discuss with your healthcare team your priorities and preferences to achieve the best possible quality of life. Increasing awareness of the importance of delivering improved quality of life as well as improved survival for cancer patients, together with major steps forward in the technology for measuring quality of life using Patient Reported Outcome Measures (PROMs), has resulted in an increasing emphasis on quality of life in cancer care. PROMs may be used in clinical trials and in everyday clinical care and can improve the quality of care and deliver better outcomes for patients, including emotional and social wellbeing. 12,13

8. You have a right to receive optimal supportive and palliative care, as relevant, during any part of your cancer journey. Palliative care addresses the symptoms and quality of life of patients. The expertise of palliative care teams is valuable for patients at all points during their cancer journey. ${ }^{2}$ The perception that palliative care only offers benefits for patients at the end of life is incorrect. Early palliative care improves patient symptoms and quality of life, reduces hospital admissions and may, in some circumstances, improve patient survival. Successful care requires close integration between oncology and palliative care services., ${ }^{8,14}$

9. You have a right to receive and discuss with your care team a clear, managed and achievable plan for your survivorship and rehabilitation. The increasing number of cancer survivors in Europe require support to ensure that they can return to as many of the activities of normal living as possible. ${ }^{6}$ Each patient should have a survivorship care plan to set out clearly the requirements of their support and follow-up. . $5,16^{-16}$

10. You have a right to be fully reintegrated into society and protected from cancer-related stigma and discrimination, so that, in so far as is possible, you can return to work and a normal life. Reintegration into normal life and the workplace is critically important. Employees should be given the best possible opportunities to return to work. Depending on a patient's ongoing recovery and/or the adverse consequences of their cancer or its treatment, this may mean that work duties might objectively need to be modified. Employers should also be encouraged to provide as smooth a transition back to work as possible for their returning employee. Returning employees should be free of discrimination as a consequence of their disease. ${ }^{2,17}$

\section{Implementation strategies}

The central themes of the code of Practice are not dissimilar to many other efforts, stretching back to the 1990s, to formulate plans to increase the quality and consistency of cancer care across Europe. Although substantial efforts by patients, patient advocates and professionals have been made to establish a programme to tackle the disparities of cancer care across Europe, there is still insufficient resolution of the differences between countries, regions, hospitals and communities..$^{1-3,6,18}$ The team of cancer patients, advocates and professionals, together with the European Cancer Organisation, are seeking to increase the chances of a real and measurable impact on the quality of care and outcomes of Europe's cancer patients.

- To promote uptake of the Code at European, national and local levels. The European Cancer Patient's Bill of Rights upon which the Code is largely based, won the European Health Award at Gastein in 2018, emphasizing the importance of patients' rights. The authors and the European Cancer Organisation have strived to ensure the maximum and most authoritative exposure of the code at a European level. The Code was launched by the European Cancer Organisation on 23 September 2020 with the support of the European 
Commissioner for Health, Stella Kyriakides, and endorsed by a wide range of leading cancer authorities for its wide dissemination. Implementation at national, regional and local levels will require further effort and a relentless commitment to the dissemination of the code to patients, advocates, cancer professionals and cancer leagues and organizations.

- A need for uptake for individual cancer types. One of the strengths of the Code is that it is generic and can apply to a patient with any cancer of any age and at any point in their journey. However, many cancers have specific dominant issues, such as the facial and bodily image impact of head and neck cancer and breast cancer, respectively, the impact on reproduction of pelvic tumours, and the neurological consequences of brain tumours. It is envisaged that the code will be retained as a core resource, but that its provisions are made meaningful and accessible for patients with all types of cancer.

- A programme for different age groups and risk categories. Different age groups may face different challenges through the diagnosis and treatment of cancer. Cancer in children presents huge challenges to the individual patient, parents and families. Teenagers and young adults are dealing with rapidly changing physical and emotional experiences and require specialized support. Adults of working age commonly face caring for a family and workplace responsibilities. Older cancer patients face anxieties about general health and ageism. All of these extensive challenges require careful adaptation and deployment of the Code.
- A training programme to build a pan-European community of expertise and shared practice. The European School of Oncology is developing a training programme for healthcare professionals and patient advocates in the knowledge and skillsets that are required to deliver improved patient outcomes, which is substantially based upon the ideas and topics presented in the European code of Cancer Practice. The intention is to create a self-sustaining and influential network of senior healthcare professionals and patient advocates who will support each other in appropriate initiatives within their countries and areas of expertise.

- A formal process of evaluation with feedback. Through the European Cancer Organisation, it should be possible to obtain feedback on patients' and healthcare professionals' opinions and comments on the code, how it can be improved and how it can be most effectively disseminated. The European Code of Cancer Practice has been successfully launched and widely supported and endorsed. However, this is only the beginning of a prolonged and determined process of dissemination and implementation across a wide range of cancer types and in different European countries. This approach will aim not only to improve patient experience and empowerment, but also to directly and indirectly influence healthcare professionals, healthcare managers and policy-makers towards implementing the code of Practice and thereby improving quality of life and enhancing survival for cancer patients. $]$
1. The European Code of Cancer Practice. Available at: www. europeancancer.org/2-standard/66-european-code-of-cancerpractice (accessed 23 May 2021)

2. Lawler M, Oliver K, Gijssels S, et al. The European code of Cancer Practice. J Cancer Policy. 2021;28:100282.

3. Lawler M, Banks I, Law K, et al. The European Cancer Patient's Bill of Rights, update and implementation 2016. ESMO Open. 2016;1:e000127

4. Peiró Pérez R, Molina Barceló A, De Lorenzo F, et al. Policy Paper on Tackling Social Inequalities in Cancer Prevention and Control for the European Population. Available at: https:// cancercontrol.eu/archived/uploads/PolicyPapers27032017/ Policy_Paper_4_Tackling.pdf, p.69-110 (accessed 23 May 2021).

5. European Society for Medical Oncology. Available at: www. esmo.org/guidelines (accessed 23 May 2021).

6. Albreht T, Kiasuwa R, Van den Bulcke M (eds). Cancer Control Joint Action European Guide on Quality Improvement in comprehensive Cancer Control. Liubliana, Slovenia: Nationa Comprehensive Cancer Control. Ljubljana, Slovenia: Nationa Institute of Public Health, 2017. hteps.//cancercontrol.eu/ archived/uploads/images/Guide/pdf/CanCon_Guide_FINAL_ Web.pdf (accessed 23 May 2021).

7. Oberst S, van Harten W, Sæter G, et al. 100 European core quality standards for cancer care and research centres. Lance Oncol. 2020;21:1009-11.

8. Velikova G, Fallowfield L, Younger J, et al. (eds). Problem Solving in Patient-centred and Integrated Cancer Care. Oxford: EBN
Health, 2018

Selby P, Popescu R, Lawler M, et al. The value and future developments of multidisciplinary team cancer care $A m$ Soc Clin Oncol Educ Book. 2019;39:332-40.

10. Albreht $T$, Amati $C$, Angelastro $A$, et al. Integrated cancer control: the case for comprehensive cancer care networks (CCCN). In: Albreht T, Kiasuwa R, Van den Bulcke M (eds). European Guide on Quality Improvement in Comprehensive Cancer Control. Ljubljana, Slovenia: National Institute of Public Health, 2017;77-104. Available at: https://cancercontrol.eu/ archived/uploads/images/Guide/pdf/CanCon_Guide_FINAL Web.pdf (accessed 23 May 2021).

11. Selby P, Liu L, Downing A, et al. How can clinical research improve European health outcomes in cancer? I Canc POl. 2019;20:100182.

12. Gilbert A, Selby P, Velikova G. Monitoring of symptoms, toxicity and functioning using patient-reported outcome measures. In: Velikova G, Fallowfield L, Younger J, Board R and Selby P (eds). Problem Solving in Patient-centred and Integrated Cancer Care. Problem Solving in Patient-centred
Oxford: EBN Health, 2018;6-13.

Oxford: EBN Health, 2018;6-13.
3. Addario B, Geissler J, Horn MK, et al. Including the patient voice in the development and implementation of patientreported outcomes in cancer clinical trials. Health Expect. 2020;23:41-51.

14. Vanbutsele $G$, Van Belle $S$, Surmont $V$, et al. The effect of early and systematic integration of palliative care in oncology on quality of life and health care use near the end of life: a randomised controlled trial. Eur J Canc. 2020;124: 186-93.

15. Albreht T, Borrás Andrés JM, Dalmas M, et al. Survivorship and rehabilitation: policy recommendations for quality improvement in cancer survivorship and rehabilitation in EU member states. In: Albreht T, Kiasuwa R and Van den Bulcke M (eds). European Guide on Quality Improvement in Comprehensive Cancer Control. Ljubljana, Slovenia: National Institute of Public Health, 2017;135-64. Available at: https://cancercontrol.eu/ archived/ uploads/images/Guide/pdf/CanCon_Guide_FINAL_Web.pdf (accessed 23 May 2021).

16. First EORTC Cancer Survivorship Summit. Eur I Canc 2014;12:1-64

17. Braspenning I Tamminga S, Frings-Dresen $\mathrm{M}$, et al. Rehabilitation and return to work after cancer - instruments and practices. European Risk Observatory Report. European Agency for Safety and Health at Work. Luxembourg: Publications office of the European Union. Available at: https:// Publications Office of the European Union. Avallable at: https//
osha.europa.eu/en/publications/rehabilitation-and-returnosha.europa.eu/en/publications/rehabilitation-and-return-
work-after-cancer-instruments-and-practices/view (accesse work-after-cancer-instruments-and-practices/view (accessed June 2021).

18. Exarchakou A, Rachet B, Belot A, et al. Impact of national cancer policies on cancer survival trends and socioeconomic BMJ. 2018;360:k764. 